\title{
A case report of unrecognized iatrogenic bladder injury during repair of misdiagnosed sliding inguinal hernia: Tips and Tricks in the management.
}

\author{
Agu Thaddeus Chika \\ Visiting Consultant Surgeon, First Choice Specialist Hospital, Nkpor, Anambra State, Nigeria \\ Consultant Surgeon, Lecturer, Imo State University, College of Medicine, Owerri, Nigeria
}

\begin{abstract}
Injury to the urinary bladder is more likely to occur during herniorrhaphy for misdiagnosed sliding hernia. Increasing abdominal pain, abdominal swelling, hematuria and decreased urinary output soon after hernia repair need further review. Emergency resuscitation, prompt exploration and repair lead to quick recovery in this patient. This is an audit case report illustrating the need to consider sliding of the urinary bladder in huge inguinal hernia and to keep it out of harm's way during repair. It also emphasizes the need to recognize any iatrogenic injury intra-operatively, and to institute treatment in the same operative session or to take the option of early referral.
\end{abstract}

Keywords: exploratory laparotomy, herniorrhaphy, iatrogenicbladder injury, sliding inguinal hernia

Key messages

+ Sliding inguinal hernia is rare and so preoperative misdiagnosis is not unusual.

+ It is also not unusual to injure the sliding structures during herniorrhaphy.

+ However, this iatrogenic injury is preventable by adhering to the surgical principles.

+ When injury occurs, good treatment outcome starts by recognizing it intra-operatively.

+ Keeping a bladder catheter in- situ for huge inguinal hernias not only decompresses the bladder but alerts the surgeon in the event of bladder injury.

\section{Introduction}

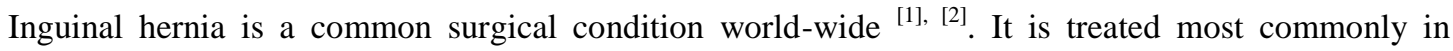
secondary health institutions and sometimes by primary health care providers in comprehensive health centers and rarely in tertiary centers in our sub-region. Misdiagnosis of sliding hernia occurs when a clinician does not observe the surgical principles during inguinal herniorrhaphy and because sliding hernia is rare, there is also the cursory attitude of treating inguinal hernias without considering the possibility of sliding. The clinician should have a high index of suspicion for sliding hernia when managing long standing huge inguinal hernia in the older males ${ }^{[1]}$.

Sliding inguinal hernia is the protrusion of a retroperitoneal organ through the inguinal canal by forming part of the wall of the sac. This is the type I sliding hernia and the commonest variety ${ }^{[2]}$. Sometimes, it is the meso-appendix or sigmoid mesentery that forms part of the wall of the sac and this is type II ${ }^{[2]}$. Type III is extremely rare and in this situation, the retroperitoneal organ herniates without a sac ${ }^{[2]}$. Sliding hernias

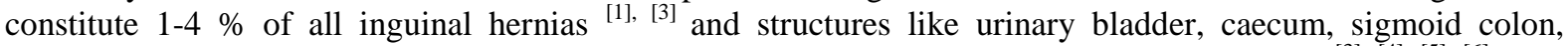
appendix, ureter, ovaries and fallopian tubes have been reported as common sliding organs ${ }^{[3], ~[4], ~[5], ~[6] . ~ T h e ~}$ problems start with mistaking any of these structures for a sac and incising it, but recognizing this injury immediately and instituting proper treatment, will impact the outcome positively.

This case highlights the need to always consider sliding hernia especially in a long standing inguinal hernia, to always adhere to the principles of inguinal herniorrhaphy and to possibly recognize and treat any iatrogenic bladder injury in the same theatre session.

\section{Presentation of case}

A 65 year old male was referred to the emergency department of our surgical facility in December 2015 with abdominal pain and swelling following a hernia repair three days previously. He also complained of scanty urine that was blood stained. The referral letter stated that the herniorrhaphy was difficult and was for a huge right inguinal hernia. The referring doctor wondered whether patient had developed acute renal shut-down.

The patient was ill-looking and the vital signs were unstable. The blood pressure was $90 / 55 \mathrm{mmHg}$, pulse was $101 / \mathrm{min}$ and weak. He was hyper-ventilating with peripheral oxygen saturation $\left(\mathrm{SpO}_{2}\right)$ of $80 \%$. Resuscitation was started with oxygen by face mask and intravenous fluid. An indwelling catheter was inserted and 30 milliliters of bloody urine was drained. A swollen, stitched right inguinal surgical wound was observed 
with fluid draining from it (Fig.1). Shifting dullness was demonstrated in the abdomen. Digital rectal examination was normal. A clinical diagnosis of iatrogenic bladder injury from herniorrhaphy was made.

The hemoglobin was $9.2 \mathrm{~g} / \mathrm{dl}$. Serum electrolytes urea and creatinine as well as blood sugar were normal. Abdominal ultrasonography showed fluid in the peritoneal cavity. We couldn't get a retrograde urethracystogram. After 8 hours of resuscitation, the patient stabilized. We planned an exploratory laparotomy with the priority search for any bladder injury. We could use the inguinal wound and extend it upwards in a right paramedian approach but, we preferred to use a virgin midline incision so that we could approach the unknown from the known.

After an informed consent and under general anesthesia, the abdominal cavity was exposed through an infra-umbilical approach, coke colored peritoneal fluid was noted. In the right side of the pelvic cavity, there was a tent on the peritoneum showing a ligature on the remnant sac and urinary bladder (Fig.2). The caecum was normal and the internal ring felt tight from the inside.

The bladder was freed and a $2.5 \mathrm{~cm}$ rent on it (Fig.3) was freshened and repaired in two layers with vicryl 0 . The Foley catheter was replaced with a three way catheter for irrigation. The inguinal wound was left alone because the extra-vasated urine would be reabsorbed. Patient recovered swiftly. The catheter was removed after 10 days and patient was discharged. A return referral was written, emphasizing the need to consider sliding of bladder in huge hernias and the need for indwelling catheter during surgery.

\section{Discussion}

Herniorrhaphy under strict adherence to the surgical principles is unlikely to be complicated by bladder injury, and worse still, go unrecognized. It is a sound surgical principle to exclude sliding of bladder in long standing huge inguinal hernias. ${ }^{[4]}$. It is equally important that a Foley catheter be inserted into the bladder to empty any part of it that may have herniated, thereby eliminating the bulge that may be mistaken for a sac. Also, hematuria is noticed in the event of bladder injury. Bladder injury in this patient following herniorrhaphy was suspected from the presentation and the referral letter. These included a huge inguinal hernia in a 65 year old male, history of hematuria and scanty urine soon after the operation, catheter drainage confirming scanty urine that was bloody without clinical evidence of acute renal shut down and fluid in the peritoneal cavity. Exploration was guided by these observations, and surgery was executed in a timely manner, which was expedient in this patient who recently had operative and anesthetic challenges and who was just barely stabilized. Keeping the stimuli for metabolic response to the minimum and modulating the response by adequate fluid therapy probably contributed to the quick recovery despite the gloomy condition of the patient on presentation.

Sliding hernia is the result of prolonged traction on the parietal peritoneum that anchors these structures to the retroperitoneal space. This is aided by prolonged increase in intra-abdominal pressure from obesity, constipation, colonic tumors and bladder outlet obstruction ${ }^{[1],[7],[8]}{ }^{[8]}$ The prevalence of sliding inguinal hernias was previously $6-8 \%{ }^{[4]}$ of all inguinal hernias but this has decreased to $1-4 \%$ in recent publications ${ }^{[3],[4],[5]}$. The reason for this is the earlier presentations of hernias in recent times. Ryan in a large population study of inguinal hernias noted a link between age of patients and duration of their hernias to the prevalence of sliding hernia ${ }^{[4]}$. The older patients signify though not invariably, the longer duration of hernias before repairs, and thus the higher incidence of sliding hernias. These are similar to the conditions in our patient. Conversely, a recent study on a large population of inguinal hernias showed a relatively younger patients and lesser duration of hernia correlating with lower prevalence of sliding hernias ${ }^{[3]}$. These reports are similar to the increase in the size of inguinal hernia into giant hernia that is often associated with sliding ${ }^{[9], ~[10]}$

Sliding hernia may not be diagnosed pre-operatively but it is absolutely necessary to do so intraoperatively. Sliding hernia has been diagnosed by visualizing a bladder stone in the hernia sac on plain radiograph ${ }^{[11]}$. Retrograde urethro-cystography is useful in suspected cases especially with urinary symptoms. During the surgery, to avoid iatrogenic bladder injury, the first thing to do is to observe the anatomy of the postero-medial wall of the sac as well as palpating for any abnormal thickness in the wall. Adhesions of organs onto the sac should be differentiated from true sliding hernias because whilst the former can be freed from the sac, the latter cannot without injuries ${ }^{[1]}$. Secondly, after identifying sliding hernia and reducing the sac's content, the conventional high sac ligation is not necessary as this may cause injury. Excision of the sac may involve the unidentified sliding structure causing injury to gut or bladder. In the former case, discharge of intestinal contents will immediately alert the surgeon but in the latter case, urine may further be mistaken for peritoneal fluid. However, an already placed bladder catheter which immediately shows hematuria would make the surgeon to recognize this injury. Furthermore, some authors had advocated that reducing the sac with its contents without ligation would eliminate the risk of injury ${ }^{[3]}$ especially for type III where it is only the retroperitoneal organ that herniates with little or no sac ${ }^{[2]}$. Finally, a low ligation ensures that the surgeon stays far from the base thereby avoiding injury to any sliding structure. 


\section{Conclusion}

Iatrogenic bladder injury could occur during repair of huge inguinal hernia especially when the surgeon does not suspect sliding and does not follow the operative principles. The success of the treatment of this injury depends on intra-operative recognition and immediate repair but when unrecognized, early referral, emergency resuscitation and exploration would improve the outcome.

\section{References}

[1]. Gespar MR, Woolley MM, Joergenson EJ. Sliding indirect Inguinal Hernia. Calif Med85(5); 1956:330-34.

[2]. Bendavid R. Sliding Hernia. Hernia 6; 2002:137-40.

[3]. Komorowski AL, Rodriquez J, Kazi R, Wysocki WM. Sliding inguinal Hernia. Int J Surg 10(4); 2012: $206-8$.

[4]. Ryan EA. Analysis of 313 consecutive cases of indirect sliding inguinal hernias. Surg GynObst 102; 1956:45-58.

[5]. Weitzenfeldt MB, Brown BT, Morillo G, Block NL. Scrotal kidney and ureter: an unusual hernia. J Urol123; 1980: 437-8.

[6]. Tahir M, Ahmed FU, Seenu V. Giant inguino-scrotal hernia: case report and management principles. Int J Surg; 6; 2008 : $495-7$.

[7]. Slater R, Amatya U, Shorthouse AJ. Colonic carcinoma presenting as strangulated inguinal hernia: report of two cases and review of the literature. Tech coloproctol. 12(3); 2008:255-8.

[8]. Tan GY, Guy RJ, Eu KW. Obstructing sigmoid cancer with local infiltration in an incarcerated inguinal hernia. ANZ J Surg; 73(2): 2003: 80-2.

[9]. Atthaphorn T, Vitoon C, Asada M, Jirawat S, Chainarong P, Thammawat P et al. Giant inguinal hernia: Report of a case and review of surgical techniques. Int J Surg Case Rep 5(11); 2014:868-72.

[10]. Agu TC, Ikeanusi MP A giant inguinoscrotal hernia in the 21 st century urban Nigeria setting? A case report and review of the literature. Niger J Gen Pract 14; 2016: 42- 5.

[11]. $\mathrm{Ng} \mathrm{ACF}$, Leung AKC, Robson WLM. Urinary bladder calculi in a sliding vesicalinguino-scrotal hernia diagnosed pre-operatively by plain abdominal radiography. AdvTher; 24;2007: 1016-9.

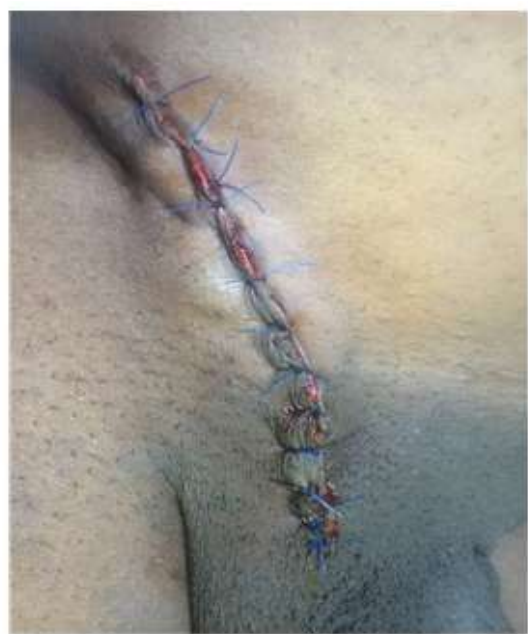

Fig.1Clinical photograph of the herniorrhaphy wound 3 days post-operationshowing swelling and extravasation of urine.

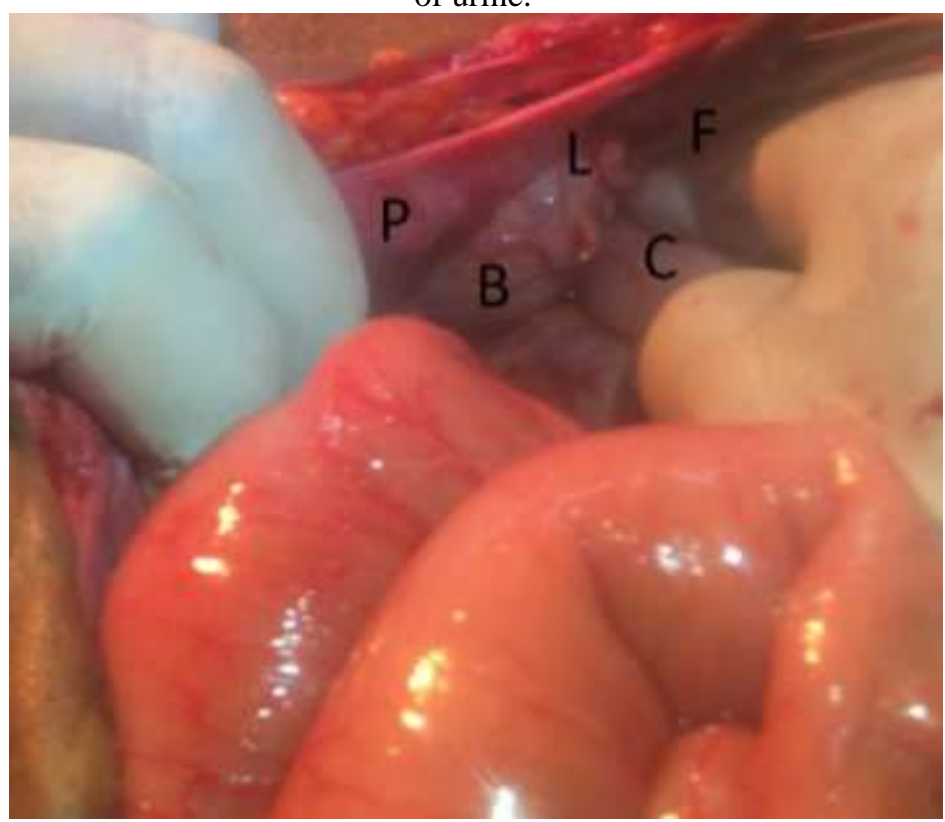


Fig.2Exploratory Laparotomy, P- peritoneum, B-bladder, L- ligature on the bladder tenting the peritoneum, Ccaecum, F- forceps pointing at the pathology.

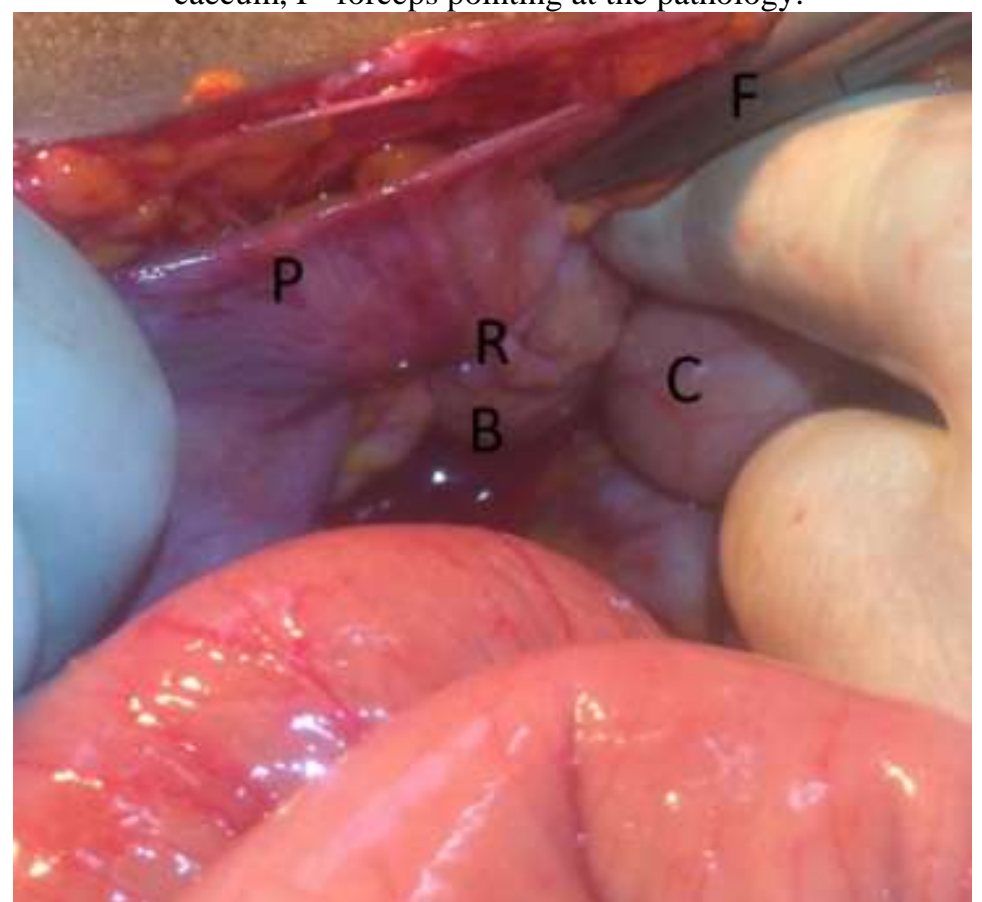

Fig.3Exploratory Laparotomy, P- peritoneum, R -rent on the bladder after removal of ligature, B- bladder, Ccaecum, F- forceps 